\title{
B cells produce pathogenic antibodies and impair recovery after spinal cord injury in mice
}

\author{
Daniel P. Ankeny, Zhen Guan, and Phillip G. Popovich
}

\author{
Center for Brain and Spinal Cord Repair, Department of Molecular Virology, Immunology, and Medical Genetics, \\ The Ohio State University Medical Center, Columbus, Ohio, USA.
}

\begin{abstract}
Traumatic injury to the mammalian spinal cord activates B cells, which culminates in the synthesis of autoantibodies. The functional significance of this immune response is unclear. Here, we show that locomotor recovery was improved and lesion pathology was reduced after spinal cord injury (SCI) in mice lacking B cells. After SCI, antibody-secreting B cells and Igs were present in the cerebrospinal fluid and/or injured spinal cord of WT mice but not mice lacking B cells. In mice with normal B cell function, large deposits of antibody and complement component $1 \mathrm{q}(\mathrm{C} 1 \mathrm{q})$ accumulated at sites of axon pathology and demyelination. Antibodies produced after SCI caused pathology, in part by activating intraspinal complement and cells bearing Fc receptors. These data indicate that B cells, through the production of antibodies, affect pathology in SCI. One or more components of this pathologic immune response could be considered as novel therapeutic targets for minimizing tissue injury and/or promoting repair after SCI.
\end{abstract}

\section{Introduction}

The consequences of neuroinflammation caused by spinal cord injury (SCI) have been inferred mostly from the results of studies that manipulate the function or survival of neutrophils, monocytes/macrophages or $\mathrm{T}$ lymphocytes ( $\mathrm{T}$ cells) (1-9). Less is known about the role played by antibody-producing B cells. In humans with SCI, elevated titers of myelin-reactive antibodies in serum and cerebrospinal fluid (CSF) suggest that SCI activates T and $B$ cells that recognize CNS proteins (10-12). Using a clinically relevant murine model of SCI, we have shown that SCI induces a long-lasting $\mathrm{B}$ cell response, characterized by enhanced lymphopoiesis in bone marrow and spleen, with increased levels of circulating IgM and IgG antibodies (13). Activated B cells also accumulate in the injured spinal cord, in which they persist indefinitely (13). Accumulation of intraspinal B cells also is associated with de novo expression of mRNA that encodes a range of autoantibodies (14).

Currently, the breadth of self/auto antigens recognized by SCIinduced antibodies is not known; however, some will bind CNS proteins and the potential exists for antibody-mediated neurodegeneration $(10,11,13)$. Previously, we showed that microinjection of sera containing SCI antibodies into the intact CNS caused focal inflammation and neurotoxicity (13). Conversely, sera from SCI B cell-knockout mice (BCKO mice), which cannot make antibodies, was innocuous (13). Collectively, these data suggest that activated B cells contribute to the pathological sequelae of SCI, presumably via production of autoantibodies and activation of downstream inflammatory cascades. Here, we prove there is a causal role for B cells as effectors of post-SCI pathology. Specifically, we show that behavioral and anatomical indices of recovery from SCI are improved in BCKO mice and that B cell-mediated pathology is caused by the antibodies they produce. Indeed, antibodies purified from SCI mice cause axon and myelin pathology with transient impairment of motor function. Antibody-mediated pathology is dependent on activation of complement and cells bearing Fc-receptors in the spinal cord. Collectively, these data suggest that con-

Conflict of interest: The authors have declared that no conflict of interest exists. Citation for this article: J. Clin. Invest. 119:2990-2999 (2009). doi:10.1172/JCI39780. trolled inhibition of B cells or plasmapheresis (plasma exchange) should be considered as therapeutic options for treating SCI.

\section{Results}

$B$ cells impair spontaneous recovery of locomotor function after SCI. Mice with and without B cells received a SCI, and locomotor recovery was evaluated for up to 9 weeks (Figure 1A). Locomotor recovery plateaued in WT mice after 2 weeks, with $35 \%$ ( $n=6$ of 17) achieving forelimb-hind limb coordination by 63 days after injury (dpi). Conversely, more than $80 \%(n=13$ of 16$)$ of BCKO mice recovered bilateral weight-supported stepping within 1 week, with additional recovery evident over the remaining 8 weeks. Ultimately, $88 \%(n=14$ of $16 ; P<0.01$ vs. WT mice) of BCKO mice recovered coordination, with $41 \%$ ( $n=7$ of 16$)$ being nearly indistinguishable from uninjured mice; only subtle deficits in control of trunk or tail were visible. Refined aspects of hind limb usage also were improved, with $\mathrm{BCKO}$ mice showing increased frequencies of forelimb-hind limb coordination, increased trunk stability, and less medial or lateral rotation of the paws during the step cycle (Figure 1B).

Spinal cord pathology is reduced in mice lacking $B$ cells. The lesion pathology caused by spinal cord contusion is characterized by a centralized core region with complete cell loss (frank lesion) and surrounding areas extending rostral and caudal to the impact site. Thus, unbiased stereology was used to quantify the volume of lesioned spinal cord at 9 weeks after injury. In BCKO mice, lesion volume was decreased more than $30 \%$ relative to SCI WT mice (Figure 2A). This was accompanied by an increase in the total volume of spared gray matter and white matter in BCKO mice (Figure 2, B-E), exceeding that in WT animals by more than $36 \%$ and $20 \%$, respectively. These differences were greatest in sections proximal/distal to the impact site (Supplemental Figure 1; supplemental material available online with this article; doi:10.1172/ JCI39780DS1). Quantitatively larger regions devoid of myelin basic protein (MBP) and axons indicated exacerbated white matter pathology in WT mice (Figure 2, F and G).

Antibody-secreting B cells (plasma cells) and antibodies accumulate in CSF and injured spinal cord. In people with SCI, high levels of antibodies are found in CSF (10-12). The functional significance of 

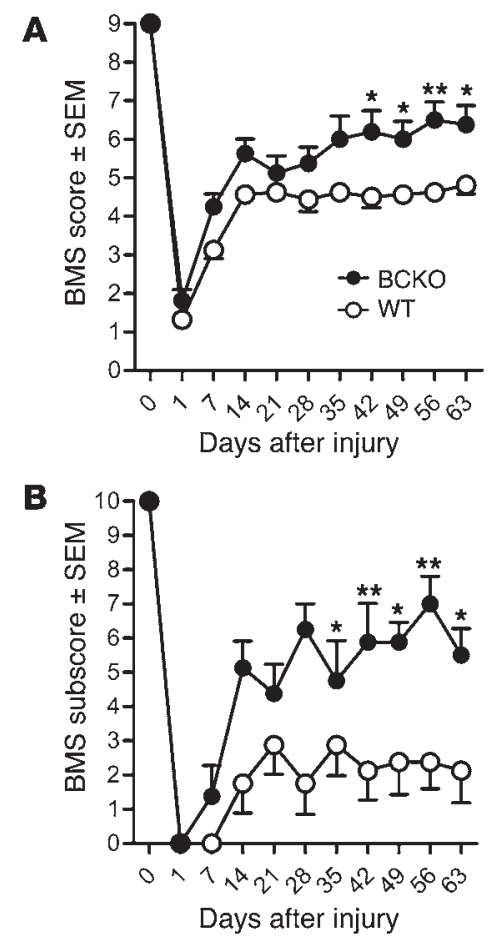

these changes is unclear; however, both physiological and pathological consequences have been proposed $(10,13)$. Here, we show for what we believe to be the first time that like that in humans with SCI, Igs (total IgM and IgG) were present in CSF of SCI WT mice but not BCKO mice (Figure 3A). To determine whether intraspinal $\mathrm{B}$ cell and antibody accumulation after SCI is unique to BL6 mice, we analyzed injured spinal cord sections from different mouse strains with distinct immunological responses to SCI, including C57BL/6 (WT mice), BALB/c, C57BL/10, B10.PL, and BCKO mice (Figure 3, B and C) (1). Staining with anti-B220, to mark mature $\mathrm{B}$ cells, revealed that the presence of intraspinal B cells infiltrated in all strains examined (Figure 3B). As we have described previously, these B cells form "follicle-like" structures around large blood vessels and are comprised of mix of T and B cells interspersed with phagocytic microglia/macrophages (data not shown; see refs. 10, 13). To test the hypothesis that antibodies directly bind antigens in the injured spinal parenchyma, we stained injured spinal cord sections with $\mathrm{F}\left(\mathrm{ab}^{\prime}\right)_{2}$ fragments of goat anti-mouse IgG (Figure 3, C and D). This allows visualization of antibodies and IgG-expressing $B$ cells without nonspecific labeling of cells expressing Fc receptors (FcRs) (e.g., microglia/macrophages). Sections from uninjured or SCI BCKO mice had negligible IgG-labeling (Figure 3, C and D). In contrast, intralesion antibody staining increased in all SCI mice as a function of time after injury (Figure 3, C and D), indicating that SCI-induced activation of $\mathrm{B}$ cells, with enhanced antibody synthesis, is not strain specific.

Levels of circulating (serum) IgM and IgG antibodies are increased in chronic SCI mice (Supplemental Figure 2 and ref. 13). Circulating antibodies were also increased after SCI in rats (Supplemental Figure 2), indicating that SCI-induced B cell responses are not species specific. Circulating Igs could cross the damaged blood-brain barrier early after SCI, or they could accumulate in the CSF and spinal parenchyma via transcytosis (15). Moreover, the progressive increase in intraspinal antibody (Figure 3C), in the face of decreas-

\section{Figure 1}

Recovery from $\mathrm{SCl}$ is improved in mice that are $\mathrm{BCKO}$ and incapable of antibody production. Locomotor function was analyzed using BMS (A) and subscore (B) analyses. The main BMS score reveals general quadrupedal locomotor ability, while the subscore reveals differences in fine locomotor control (e.g., stepping frequencies, percentage forelimb-hind limb coordination, ability to execute stepping without medial or lateral paw rotation, relative trunk stability and tail position). $n=16-17 /$ group from 2 replicate studies giving equivalent results. ${ }^{\star} P<0.05,{ }^{*} P<0.01$, versus WT; 2-way ANOVA with repeated measures, Bonferroni post-test.

ing blood-brain barrier permeability $(16,17)$, suggests that terminally differentiated, antibody-secreting plasma cells may also populate the injured spinal cord. To test this hypothesis, we analyzed the maturation state of intraspinal B cells. In all mouse strains, B cells infiltrate the lesion site, in which they form dense cell clusters (13) (Figure 3, B and E). Most $\mathrm{B}$ cells are $\mathrm{B}_{22} 20^{+}$and $\mathrm{IgG}^{+}$, indicting that they are activated but have not differentiated into antibody-secreting plasmVa cells (Figure 3E). However, terminally differentiated B220- plasma cells with intense, cytoplasmic IgG-labeling were prevalent in SCI lesions (shown for BL/6 mice; Figure 3F).

SCI-induced antibodies cause behavioral deficits in naive mice. Sera from SCI WT but not SCI BCKO or uninjured mice causes neuroinflammation and neuron death when microinjected into intact CNS (13). Antibodies, cytokines, or other blood-derived factors produced after SCI could cause these changes. Since high levels of antibodies exist in the circulation and CSF after SCI, we tested whether these by-products of B cell activation could directly cause the pathology that we described previously. If so, this would implicate antibodies in post-SCI neurodegeneration.

Antibodies were purified from blood of control or SCI mice (see Supplemental Figure 3). Purified antibodies were microinjected into the spinal cord of naive mice at a concentration of $\sim 0.6 \mu \mathrm{g} / \mu \mathrm{l}$, which is approximately one-tenth the concentration of IgG in normal blood $(13,18)$. Overground locomotor function was monitored in all mice for up to 7 days. Mice that were microinjected with antibodies from control mice $(n=9)$ had no obvious gait deficits at any time after injection (Figure 4, A and C; Supplemental Video 1). In contrast, the hind limb ipsilateral to the injection site became paralyzed in all mice injected with SCI antibodies $(n=6$, Figure 4, B and C; Supplemental Video 2). Full paralysis was evident during the first 48 hours, with varying degrees of recovery noted over the course of 1 week. Even by 1 week, mild to substantial hind limb deficits persisted (Supplemental Video 3). Some mild functional impairment (e.g., dorsal stepping, toe drags) was visible initially in the contralateral hind limb, but normal function was restored within 72 hours (data not shown).

Anatomical analyses of neurotoxic SCI-induced antibodies. No visible pathology was present in spinal cords injected with control antibodies (Figure 4D). In contrast, large necrotic inflammatory lesions occupied the ipsilateral gray matter and most of the white matter in all mice injected with SCI antibodies (Figure 4, E and F). Of note was the complete loss of neurons over a rostro-caudal distance of approximately $3.0 \mathrm{~mm}$, with some pathology evident across the midline in gray and white matter. Contralateral pathology was restricted to within approximately $2 \mathrm{~mm}$ of the injection site and likely contributed to the transient loss of function in the contralateral hind limb of some mice. In white matter on the injected side, axons were lost or extensively damaged and these 

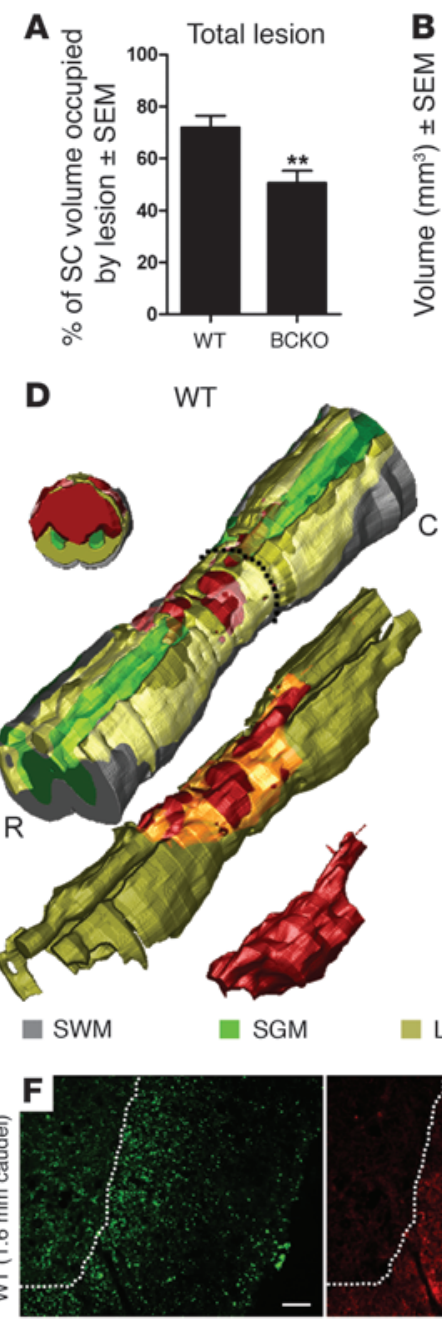

Lesioned WM
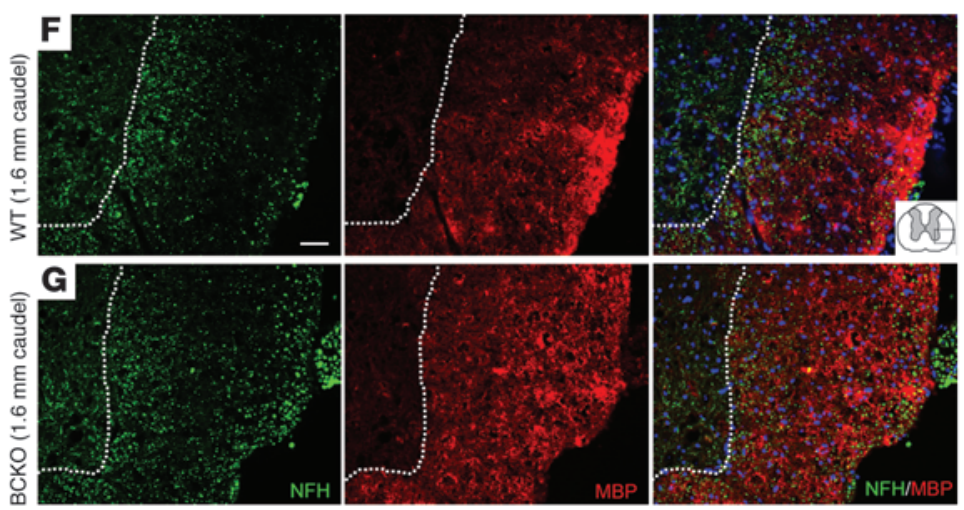

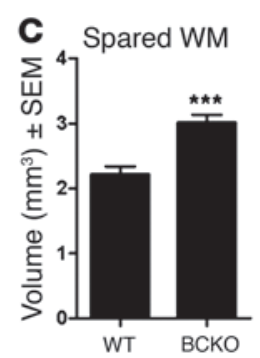

Figure 2

Significant neuroprotection is evident in the injured spinal cord of BCKO mice at $63 \mathrm{dpi}$. The total lesion volume (A) is reduced in BCKO mice and is accompanied by marked sparing of spinal cord (SC) gray matter (GM) (B), and white matter (WM) (C). Volumes were estimated using Cavalieri's method. (D and E) 3D reconstructions of spinal cords taken from animals with total lesion volume that is closest to the average for each group. Gray indicates spared white matter (SWM; regions containing myelin and axon profiles that are morphologically normal); green indicates spared gray matter (SGM); red indicates frank lesion (complete loss of normal cytoarchitecture); and yellow indicates lesioned white matter (regions where axons and myelin are absent). Coronal slabs are sampled at 0.8-mm caudal to the injury epicenter and are marked by dashes in the complete $3 D$ reconstructions. C, caudal; R, rostral. Immunofluorescent double labeling of spared white matter 1.6- $\mathrm{mm}$ caudal to the injury epicenter from a WT $(\mathbf{F})$ and BCKO mouse $(\mathbf{G})$ reveals increased sparing of axons (green, anti-NFH) and myelin (red, anti-MBP) in BCKO mice. Dotted line delineates gray matter-white matter interface. Blue (DAPI) staining in merged image reveals cell nuclei. Cartoon in top right panel depicts imaged region. Scale bar: $0.5 \mathrm{~mm}(\mathbf{D}$ and $\mathbf{E}) ; 40 \mu \mathrm{m}$ (F and G). ${ }^{*} P<0.05,{ }^{* *} P<0.01,{ }^{* * *} P<0.001$ versus WT; 2-tailed $t$ tests. All data was collected at $63 \mathrm{dpi}$. regions coincided with intense microglia/macrophage activation (Figure 4, G-I). Few T cells infiltrated sites of antibody-mediated pathology (data not shown).

SCI-Igs cause neuropathology via complement-and FcR-dependent mechanisms. When antibodies bind antigen, they form immune complexes (ICs) that cause tissue injury through activation of complement or recruitment/activation of cells bearing receptors for IgG, the FcRs (19-26). To determine whether these mechanism(s) contribute to the pathology and loss of function caused by SCI antibodies, control or SCI antibodies were injected into the intact spinal cord of mice deficient in complement component $\mathrm{C} 3\left(\mathrm{C3}^{-/-}\right)$ or the $\mathrm{FcR} \gamma$ chain $\left(\mathrm{FcRg}^{-}\right)$.

As before, SCI antibodies caused complete but transient paralysis when injected into WT mice (Figure 5A). In contrast, hind limb deficits were attenuated in $\mathrm{C3}^{-/-}$and $\mathrm{FcRg}^{-/-}$mice, and the rate of spontaneous recovery was accelerated relative to WT mice (Figure 5A). Stereological analyses of the spinal cord lesions from each mouse revealed significantly reduced pathology across the rostrocaudal axis of the spinal cord in $\mathrm{C3}^{-/-}$and $\mathrm{FcRg}^{-1}$ mice (see Figure 5, B-D, and Supplemental Figure 4).

The latter data suggest that the ICs formed after SCI may cause injury to target cells in part through activation of complement. To examine whether intraspinal antibodies overlap with complement near or on putative target cells after SCI, confocal microscopic analyses of injured WT spinal cords were completed. In WT mice (Figure 6, A, C, E, and F), antibody/C1q deposits were prevalent and consistently decorated cells with endothelial, glial, and neuron morphologies (Figure 6, E and F; endothelial labeling not shown). In contrast, minimal antibody/C1q labeling was found throughout injured BCKO spinal cords (Figure 6, B and D).

\section{Discussion}

SCI triggers immune responses that can simultaneously exacerbate tissue injury and promote CNS repair (27-29). After SCI, B cells 

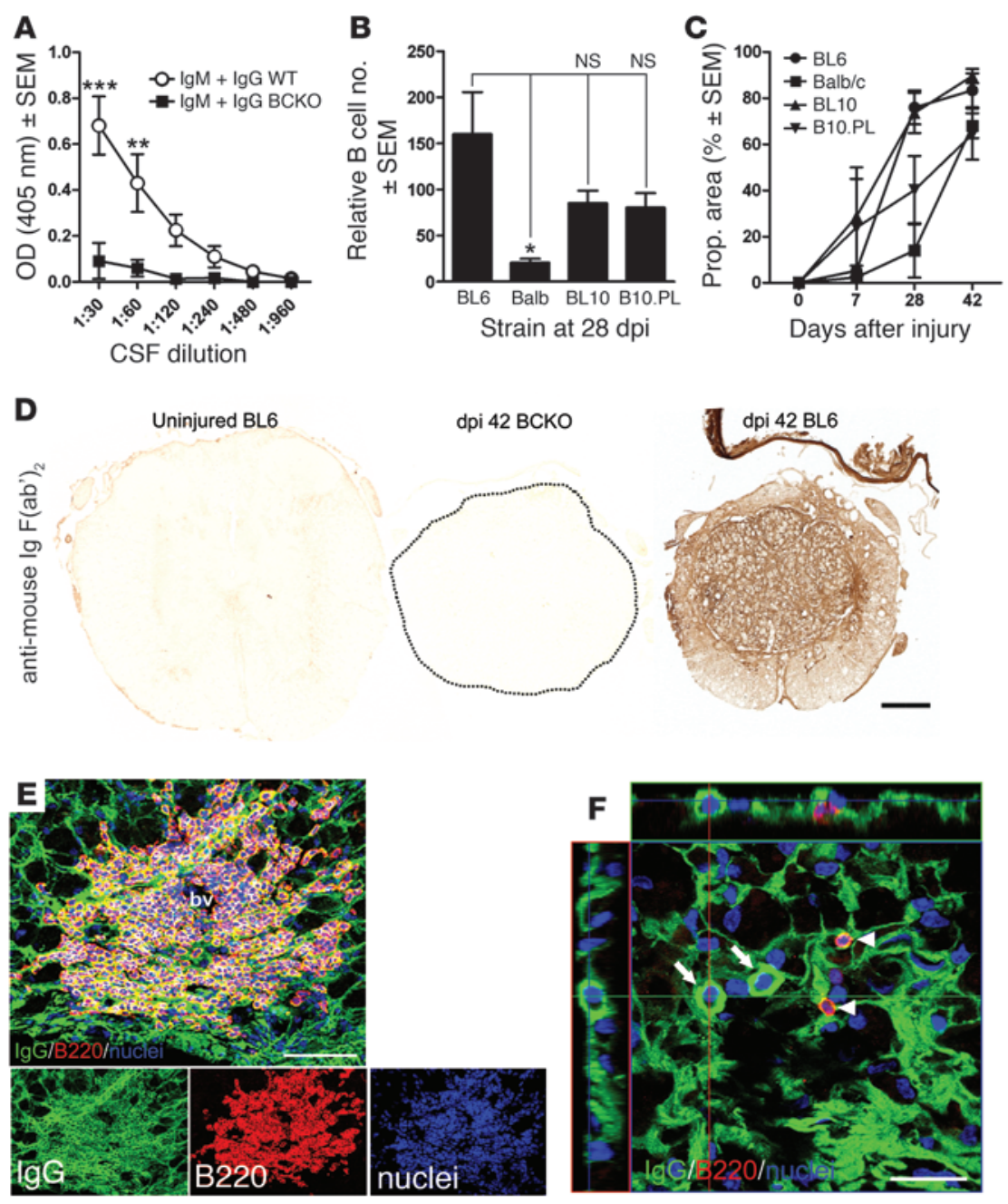

\section{Figure 3}

Antibodies, B cells, and plasma cells accumulate in CSF and injured spinal cord. (A) In contrast to WT mice, BCKO mice fail to produce intrathecal antibodies (ELISA analysis of CSF from $n=8$ WT and BCKO mice). (B and C) Quantitation of intraspinal $B$ cell accumulation at $28 \mathrm{dpi}$ (B) and the proportional (prop.) area of IgG staining as a function of time after $\mathrm{SCl}(\mathrm{C})$ at the injury site in BL/6 (WT), BALB/c, C57BL/10 (BL10), and B10.PL mice. The intraspinal accumulation of $B$ cells and antibodies is not strain specific, only the magnitude varies; $n=4-8$ mice/strain; each bar in $\mathbf{B}$ represents the average total number of $\mathrm{B} 220^{+}$lymphocyte profiles in 3 equally spaced sections (1/20th series) spanning $600 \mu \mathrm{m}$ and centered at the epicenter. (D) Representative sections from uninjured $\mathrm{BL} / 6, \mathrm{SCI} \mathrm{BCKO}$ (spinal cord circumscribed by dotted line), and WT mice (42 dpi) reveal the distribution of endogenous antibodies and the specificity of IgG labeling quantified in $\mathbf{C}$, i.e., no labeling exists in spinal cord of uninjured or BCKO mice. (E) Flattened confocal z-stack image reveals accumulation of endogenous antibodies (green, $\mathrm{Ig}$ ) and $\mathrm{Ig}^{+}$ $B$ cells in the injured spinal cord (42 dpi, individual color channels shown below). bv, blood vessel. (F) Flattened z-stack image with $\mathrm{x}, \mathrm{y}, \mathrm{z}$-projections showing $\mathrm{B}^{2} 20^{-}$plasma cells with IgG+ ${ }^{+}$cytoplasm (arrows) nearby but distinct from IgG+B220+ B cells (arrowheads). Scale bars: $200 \mu \mathrm{m}$ (D); $50 \mu \mathrm{m}$ (E, top panel); $100 \mu \mathrm{m}$ (E, bottom panels); $20 \mu \mathrm{m}(\mathbf{F}) .{ }^{* * *} P<0.001,{ }^{* \star} P<0.012$-way ANOVA with Bonferroni post-test: ${ }^{*} P<0.05$, 1-way ANOVA with Tukey’s post-test. produce antibodies that bind CNS and non-CNS antigens (10, 11, 13). Here, we show that this component of the immune response exacerbates pathology caused by SCI. Notably, in mice that are $\mathrm{B}$ cell-deficient and incapable of antibody production, lesion pathology was reduced and spontaneous recovery of locomotor function was improved. Also, injection of antibodies purified from SCI mice into naive/uninjured spinal cord caused consistent paralysis and pathology. The "sterile inflammation" and tissue injury caused by SCI antibodies resembles that described in models of muscle and gut ischemia/reperfusion injury, which like SCI, involve activation of complement C1q and cells bearing FcRs (30-32).

From our present and past data (13), it is clear that SCI potently activates $B$ cells, culminating with increased synthesis of autoantibodies. Our laboratory also has shown a seemingly paradoxical effect of SCI on B cell function, i.e., complete spinal cord transection at high thoracic levels (T3) induces marked apoptosis of splenic B cells within 72 hours after injury (33). Experimental immunizations during this acute postinjury interval fail to elicit antibody synthesis (33). This phenomenon appears to be level dependent as neither complete spinal transection nor incomplete spinal contusion injury at mid-thoracic level (T9) cause significant B cell apoptosis (33). On the contrary, T9 spinal contusion activates B cells within 24 hours after injury, causing them to secrete IgM and then IgG antibodies. Then, after approximately 14 days, substantial levels of autoantibodies are detected in T9 SCI mice. We have not evaluated the impact of T3 SCI on autoantibody synthesis. However, despite the rapid onset of acute immune suppression in this model, it would not be surprising if $B$ cell function, including synthesis of autoantibodies, was restored at later times after injury. Indeed, although injuries at cervical or lower spinal levels in humans cause immune suppression, high titers of CNS-reactive autoantibodies also exist in these individuals (10-12). Additional research is needed to determine how the level or severity of a SCI influences the kinetics and magnitude of B cell activation and autoantibody synthesis. Additional functions of B cells, including their potential role as antigen presenting cells or immune-regulatory cells, should also be considered as these functions may predominate during the acute phase of injury ( $<14$ days after injury), i.e., before significant production of autoantibodies is detectable.

We predict that delayed accumulation of intraspinal antibodies causes pathology, in part by activating complement. C1q binds antigen-antibody (immune) complexes, and this initiates enzymatic conversion of other complement proteins. The result is the formation of a lytic membrane attack complex and recruitment/ activation of myeloid lineage cells (e.g., microglia/ macrophages) 

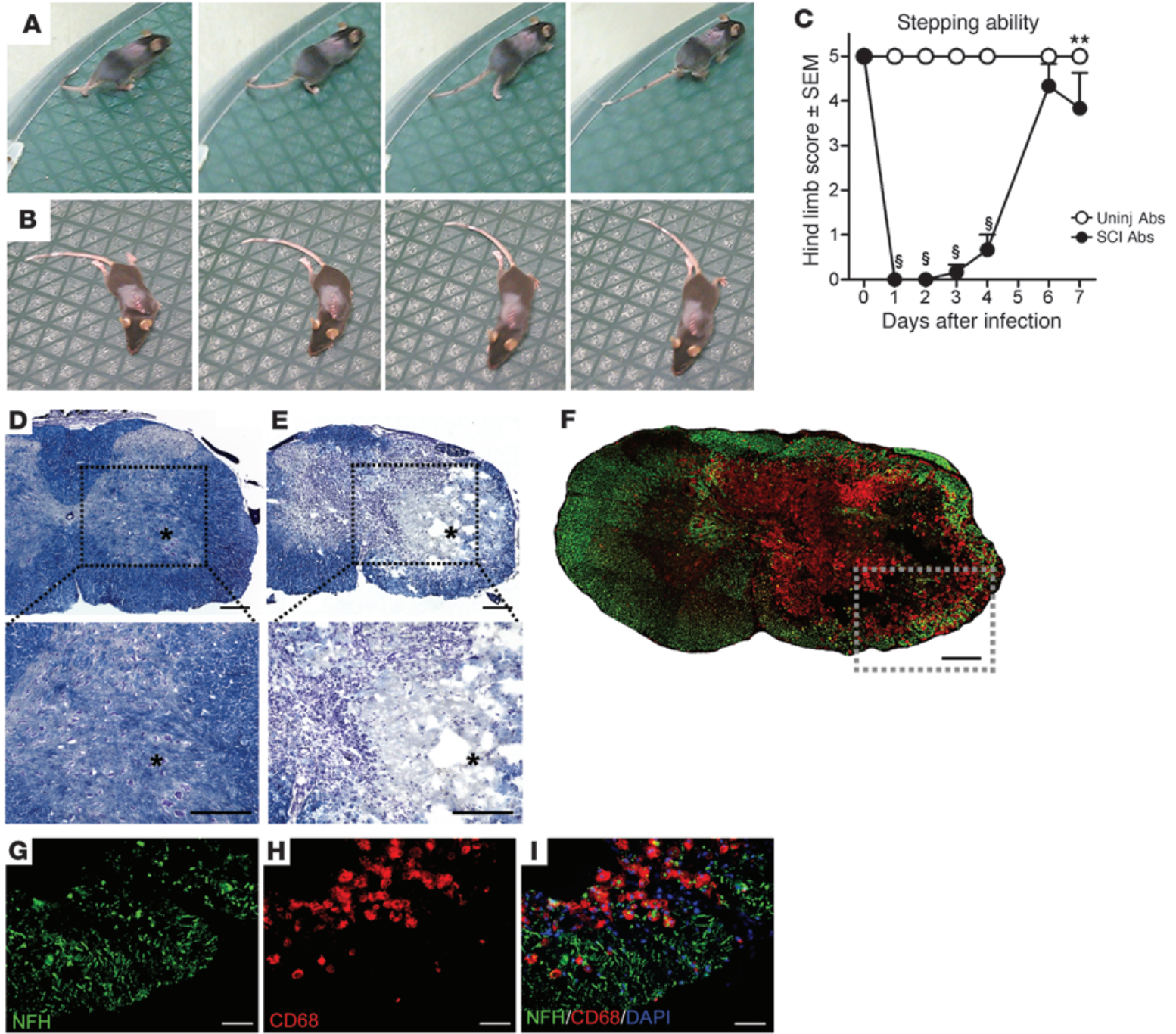

\section{Figure 4}

Unilateral intraspinal microinjection of antibodies purified from SCI mice causes hind limb paralysis and neuropathology. (A and B) A sequence of still video images 1 day after injecting naive (uninjured) mice with control (uninjured) (A) or SCl antibodies (B). One complete step cycle is depicted in both cases. (C) Summary of hind limb function ipsilateral to the site of injection. Scoring is based on the BMS scale (0-5): 0, complete paralysis; 5 , plantar stepping during more than $50 \%$ of step cycles. Scores above 5 were not considered, as our analyses were restricted to the limb on the injected side only. uninj, uninjured mice. ${ }^{* \star} P<0.01$ versus uninjured; $\$ P<0.001$ versus uninjured, 2 -way ANOVA with Bonferroni's post-hoc test. (D and E) Low- and high-power images from a mouse injected with control (D) or SCl antibodies (E), respectively. Note that intraspinal pathology is only evident in mice receiving $\mathrm{SCl}$ antibodies; the asterisk indicates the injection target. (F) Phagocytic microglia/ macrophages (red, anti-CD68) colocalize with axon/neuron pathology (green, anti-200-kDa NFH) at the site of injection in mice receiving SCI antibodies. (G-I) High-power images of boxed region in F. Scale bars: $0.2 \mathrm{~mm}(\mathbf{D}-\mathbf{F}) ; 50 \mu \mathrm{m}(\mathbf{G}-\mathbf{I})$.

that bear complement receptors. Using a model of spinal contusion injury, we observed marked neuroprotection in mice that could not make ICs (i.e., BCKO mice); these mice had minimal C1q deposition at/nearby sites of SCI. Conversely, in SCI WT mice, intraspinal antibody deposits were found in conjunction with $\mathrm{C} 1 \mathrm{q}$ labeling. Recently, a deficiency in C1q was shown to be neuroprotective and promote functional recovery after SCI or traumatic brain injury (34-36). C3 deficiency also confers neuroprotection with reduced inflammation and improved functional recovery after SCI (37). Further support for our hypothesis comes from our studies showing that injection of pathogenic SCI antibodies into the spinal cord of complement-deficient mice is benign, unlike identical injections into WT spinal cord. Injection of SCI antibodies into FcR-deficient mice produced similarly mild injuries, suggesting that $\mathrm{SCI}$ antibodies also initiate inflammation by ligating FcRs on macrophages, microglia, or other FcR-bearing immune cells $(19-26,38,39)$. It is intriguing that despite the delayed and chronic accumulation of intraspinal B cell clusters and autoantibodies, a decline in locomotor function was not observed at later times after injury in WT mice (see Figure 1A). Instead, functional recovery plateaued. This could indicate that antibodies are antagonizing mechanisms of endogenous repair rather than causing direct toxicity to cells that are unaffected by the primary trauma. For example, we have preliminary evidence that autoantibodies 
A
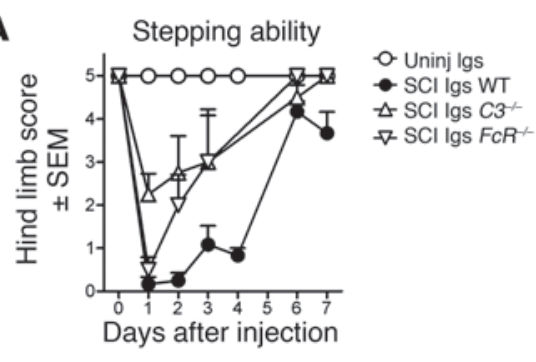

B
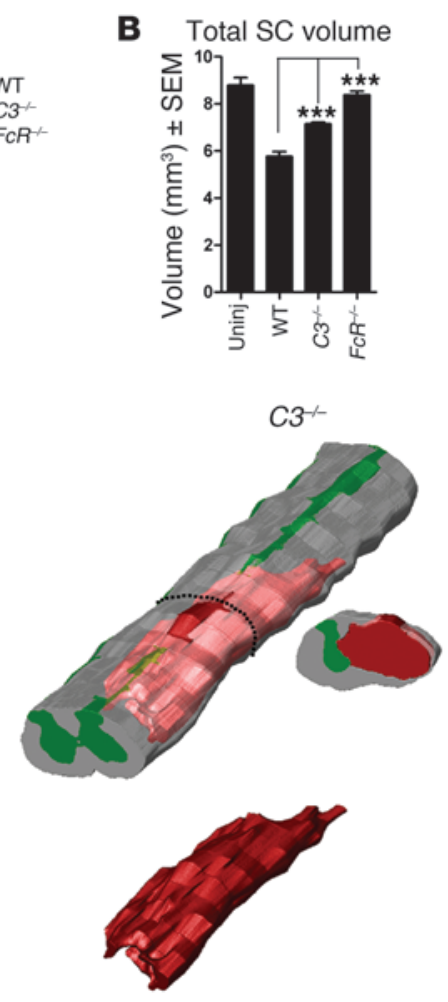
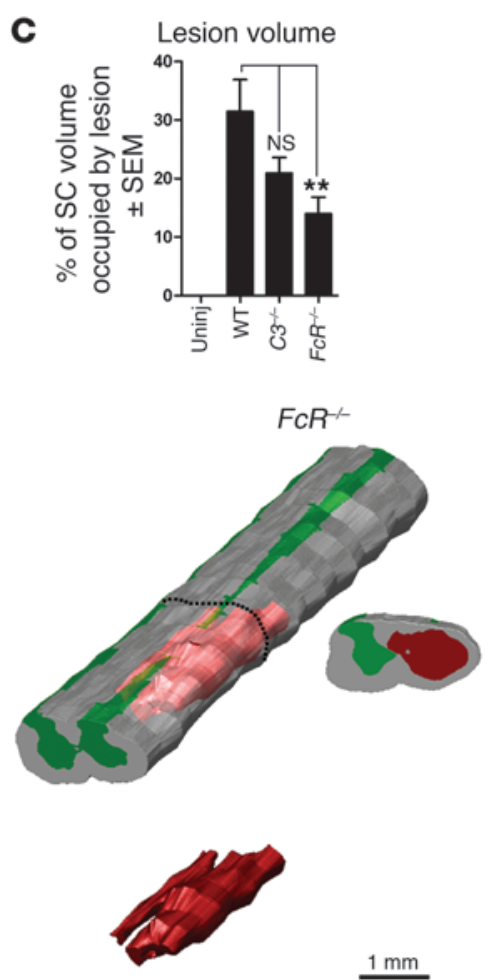

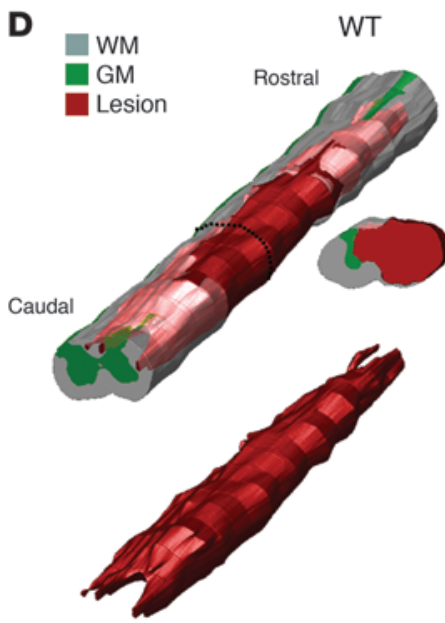

wT 

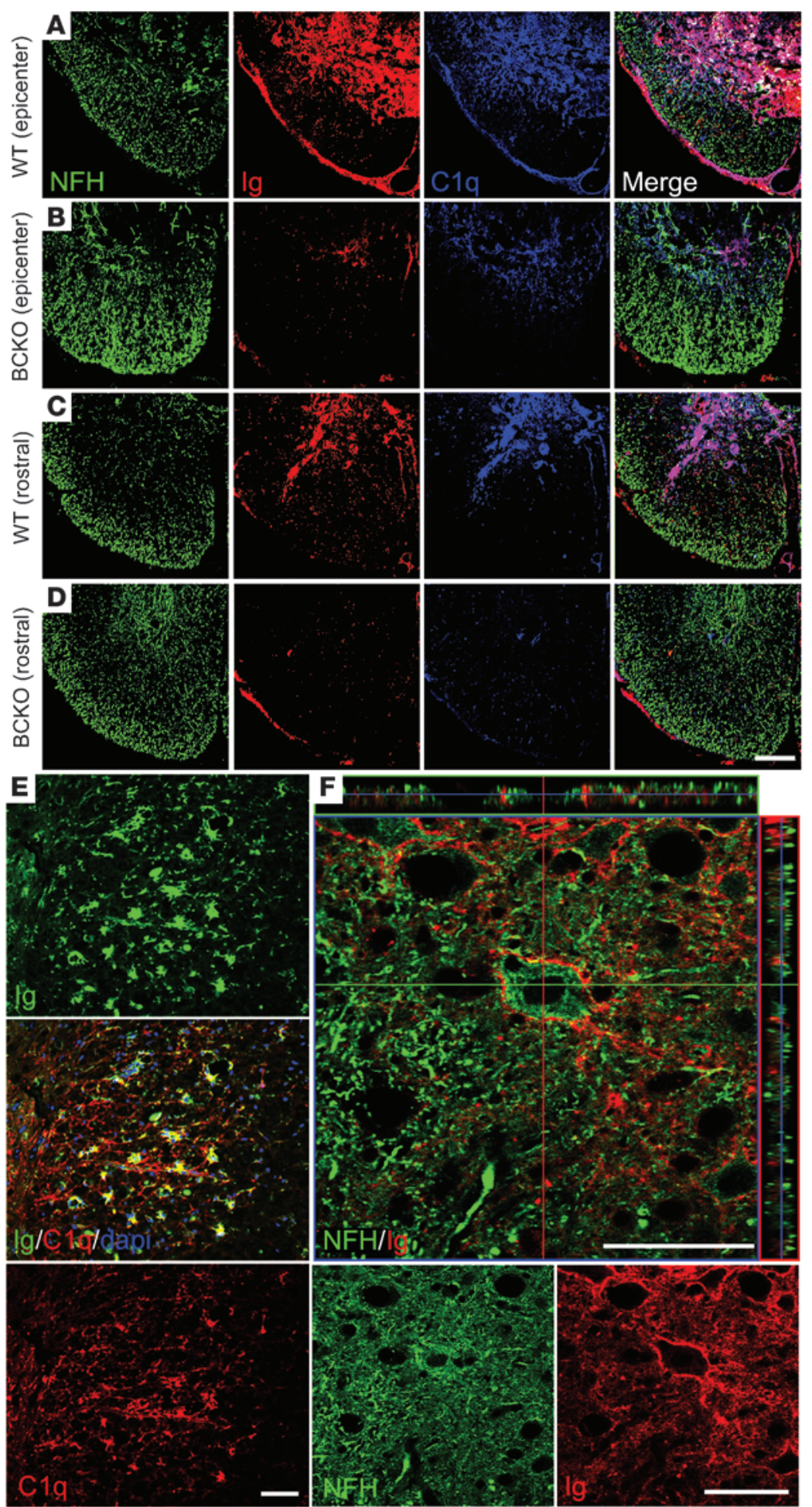

Figure 6

IgG and complement $\mathrm{C} 1 \mathrm{q}$ colocalize in regions of pathology in spinal cord of WT mice. (A and C) Confocal microscopy reveals a relationship among axons (green, anti-200-kDa NFH), Igs (red, anti-mouse Ig), and complement $\mathrm{C} 1 \mathrm{q}$ (blue, anti-C1q) in the ventrolateral funiculus at a site and rostral $(1.6 \mathrm{~mm})$ to a site of $\mathrm{SCl}$ in WT mice. (B and $\mathbf{D}$ ) In BCKO mice, sparse Ig and $\mathrm{C} 1 \mathrm{q}$ labeling can be seen among markedly preserved axon tracts and gray matter. (E) Colocalization of IgG (green) and C1q on cells with glial morphology in the lateral funiculus approximately $400-\mu \mathrm{m}$ caudal to the epicenter. (F) $x / y / z$-projections of a flattened z-stack image from a section adjacent to site of injury, showing IgG and NFH colocalization in the ventral horn, on a cell with motor neuron morphology (center): single channel images are depicted below. Scale bars: $100 \mu \mathrm{m}$ (A-D); $50 \mu \mathrm{m}$ (E and F).

microglia and macrophages, including the induction of recycling endocytosis and TNF- $\alpha$ release, ligation of antiinflammatory FcRs, and induction of remyelination via stimulation of oligodendrocyte progenitor cells (50-54). These functions predominate when antibody concentrations are low or when antibodies exist in monomeric form, i.e., not bound to antigen (50). Presumably, these mechanisms underlie the therapeutic benefits imparted by intravenous Ig therapy for CNS and non-CNS diseases $(51,55,56)$. However, as antibody levels rise and parallel inflammatory cascades are initiated, antibodies cause pathology; even monomeric IgG becomes pathologic at high concentrations (50). This would explain why BCKO mice show progressive functional recovery, with reduced pathology at times when B cell activation and antibody synthesis appear to limit recovery levels in WT mice. Since the sterile inflammation and tissue destruction caused by intraspinal injection of SCI antibodies was mitigated in complement or FcRdeficient mice, we can conclude that these mechanisms of immunity are activated downstream of B cell activation and antibody synthesis after SCI. This hypothesis is supported by the observation that $\mathrm{IgG} / \mathrm{C} 1 \mathrm{q}$ deposits are found in and near sites of tissue injury, including areas of visible neuron pathology, and functional recovery is improved in complement-deficient mice (35).

Another interesting but unproven explanation for our results is that the postinjury rise in circulating and CSF antibodies is a mechanism of protein homeostasis (57). In acute poststreptococcal glomerulonephritis, circulating IgG levels rise precipitously but without a proportional change in IC for-

CNS. Revealing the identity and source of these antibodies is of utmost importance, since it is clear that not all antibodies are pathogenic and even those with this capacity may trigger repair at lower concentrations. Indeed, antibodies purified from naive or sham-injured mice are benign when they are injected into normal spinal cord or brain. Natural autoantibodies exist in these preparations and likely help coordinate neuroprotective functions in mation in kidney. This is intriguing since pathogenic antibodies cause kidney pathology in this disease. There is speculation that high titers of IgG help "buffer" pathogenic proteins, including antibodies, while minimizing the loss or degradation of proteins (e.g., albumin) that are depleted during the course of the disease.

In summary, the present data reveal an unexpected role for $B$ cells and antibodies as effectors of pathology after SCI. Presum- 
ably, various self antigens are released or become altered by SCI, causing B cell activation and secretion of antibodies that trigger pathogenic complement cascades and microglia/macrophages. Future studies should determine whether this innate pathological cascade can be titrated to evoke repair and whether acute $\mathrm{B}$ cell-depletion therapies that are in use for other indications (e.g., Rituxan) could be effective in promoting recovery from SCI.

\section{Methods}

\section{Mice}

Specific pathogen-free C57BL/6J (WT, $n=28$ ), IgH-6 (BCKO, $n=17$; BL6 background), and B6.129S4-C3tm1Crr/J $\left(\mathrm{C}^{-/-}, n=4\right)$ mice were obtained from The Jackson Laboratory. $\mathrm{FcRg}^{-/}$mice $(n=6)$ were obtained from Taconic Farms. C57BL/10, B10.PL and BALB/cJ mice were from a previous study (1) and were obtained from Harlan Laboratories. BCKO mice are phenotypically similar to WT mice, except that deletion of surface IgM arrests B cell maturation at an early (pre-B cell) stage, and mice therefore do not express functional B cells (58). All mice were females, age 7-8 weeks old and weighed 17-22 $\mathrm{g}$ at the time of surgery. All mice were housed in HEPA-filtered Bio-clean units in a sterile room (barrier housing). All procedures were approved by and performed in accordance with The Ohio State University's Institutional Lab Animal Care and Use Committee.

\section{$S C I$}

Mice received a mid-thoracic spinal contusion injury, using The Ohio State University electromechanical spinal contusion device, as described previously $(3,27,59)$. Briefly, mice were anesthetized with ketamine and xylazine $(80 \mathrm{mg} / \mathrm{kg}$ and $10 \mathrm{mg} / \mathrm{kg}$, respectively, i.p.) and then given prophylactic antibiotics (1 mg/kg, subcutaneous; Gentocin). Using aseptic technique, a partial vertebral laminectomy was performed at the mid-thoracic level (T9-10). The exposed dorsal spinal surface (T9 spinal level) was displaced a calibrated vertical distance $(0.5 \mathrm{~mm}$ over $\sim 25 \mathrm{~ms})$, producing a moderately severe SCI. After surgery, muscles and skin were sutured, and mice were hydrated with physiological saline ( $2 \mathrm{ml}$, subcutaneous). Bladders were voided manually 2 times a day, and hydration was monitored daily, and urinary $\mathrm{pH}$ was monitored weekly. All animals were within normal parameters of displacement, force, and impulse-momentum (Grubb's test to detect outliers; $t$ test comparing biomechanic parameters among groups or individual strains yielded $P$ values of more than 0.72 for all measures).

\section{Behavioral analysis}

Locomotor recovery was compared using the BMS locomotor rating scale, specifically designed for use in SCI mice (60). The BMS is a 10-point scale based on operational definitions of hind limb movement, with additional emphasis placed on evaluating trunk stability. Briefly, individual mice were simultaneously observed by 2 investigators for a 4-minute testing period, during which hind limb movements, trunk/tail stability, and forelimbhindlimb coordination were assessed then graded according to published methods (60). The subscore component of the BMS scores individual aspects of fine locomotor control in the hind limbs (e.g., paw rotation at initial contact) and trunk/tail (e.g., ability to maintain the tail in an upright position during locomotion), i.e., aspects of locomotion that if they were to change alone, would not necessarily change the overall BMS score (60). For this reason, subscoring is a useful adjunct to the BMS scale and helps reveal more subtle changes in behavioral recovery.

\section{CSF collections}

CSF was collected from anesthetized mice at $63 \mathrm{dpi}$ via the cisterna magna. Briefly, a sharpened $0.5-\mu l$ Hamilton syringe was used to puncture and then aspirate $\sim 10 \mu \mathrm{l}$ of clear CSF. Mice were then immediately perfused as outlined below. CSF samples were transferred to individual tubes and frozen at $-80^{\circ} \mathrm{C}$. Total Ig levels were measured by ELISA, as described previously (13).

\section{Antibody microinjection studies}

Serum collection. Whole blood was obtained from awake, unrestrained mice via retro-orbital puncture, as described previously (13). Samples were collected from SCI mice before injury and at 42 days after SCI and from uninjured mice at 42 days after laminectomy (see below).

Purification of antibodies from SCI and uninjured serum. Total serum IgM and IgG were purified in a multistep procedure, using products from Thermo Scientific (Pierce). Sera samples ( $n=10 \mathrm{SCI}, n=12$ uninjured; $30-60 \mu \mathrm{l}$ / mouse) were randomly selected and pooled to yield $510 \mu$ l total serum per group, $10 \mu \mathrm{l}$ of which was reserved for determination of original Ig "purity" and concentration (see below). The remaining $500 \mu \mathrm{l}$ samples were subjected to ammonium sulfate precipitation by adding $0.5 \mathrm{ml}$ ice-cold saturated ammonium sulfate dropwise over a 15 -minute period. Proteins were allowed to precipitate overnight at $4^{\circ} \mathrm{C}$ and then were spun at $3,000 \mathrm{~g}$ for 30 minutes, and supernatants (including soluble protein contaminants) were discarded. Precipitated proteins (including $\operatorname{IgG}$ and $\operatorname{IgM}$ ) were resuspended in $0.5 \mathrm{ml}$ Melon Gel Purification buffer, $\mathrm{pH} 7.0$ (ThermoScientific), then residual ammonium sulfate was removed by dialyzing in four $400-\mathrm{ml}$ volumes of the same buffer. Dialysis was performed in 20-kDa molecular weight cut-off Slide-A-Lyzer Dialysis Cassettes (Thermo, Pierce), with continuous agitation via a stir bar. Dialysis buffer was completely replaced 3 times, with the time in each volume being 1 hour, 2 hours, 14 hours, and then 1 hour. After dialysis, an additional $10 \mu \mathrm{l}$ volume was set aside for testing (see below), and the remaining volume was subjected to IgG purification using the Melon Gel Serum IgG Purification Kit (Thermo, Pierce, according to the manufacturer's instructions). After completion of the Melon Gel procedure, IgM was purified by eluting bound proteins from the Melon Gel support columns, then passing the remaining non-IgG "contaminants" through centrifugal filtration columns with a $100-\mathrm{kDa}$ molecular-weight cut-off. Purified IgG and IgM were combined and again filtered/concentrated in centrifugal filtration columns (100-kDa molecular-weight cut-off). The retentate was resuspended in Melon gel purification buffer ( $\mathrm{pH} 7.2)$ to identical concentrations $(0.59 \mathrm{mg}$ total $\operatorname{IgM}+\operatorname{IgG} / \mathrm{ml})$ and stored at $4{ }^{\circ} \mathrm{C}$ until used for injection (within 5 days). Resulting samples were evaluated for Ig purity and concentration by SDS-PAGE/Western blot and ELISA, respectively (see below and Supplemental Figure 3).

Verification of antibody purity. Verification of antibody purity was determined by SDS-PAGE and Western blotting. For electrophoresis, $10 \mu \mathrm{g}$ $(1.69 \mu \mathrm{l})$ total purified protein and equal volumes of pooled, unpurified sera were used. Samples were separated by SDS-PAGE on $12 \%$ Bis-Tris gels (Invitrogen) and then transferred to nitrocellulose membranes (parallel gels were run identically but total proteins were stained in-gel using Coomassie's reagent). Immediately after transfer, membranes were stained for total proteins with Ponceau S, destained, then digitally photographed to visualize protein content. After blocking, membranes were probed with HRP-conjugated anti-mouse Ig (heavy and light chain antibody, which detects IgM, IgG, and IgA). Bound Abs were detected by chemiluminescence (Millipore Immobilon Western HRP Substrate) followed by exposure to autoradiography film (Kodak Biomax).

Determination of antibody concentration in purified samples. Concentration of total $\operatorname{Ig}$ (IgG and IgM) was determined using ELISA, as described previously (13). Briefly, purified antibodies were diluted 1:100 in purification buffer, and quadruplicate samples were compared to a standard dilution series of purified mouse IgG1 (Southern Biotech).

Unilateral antibody microinjections. Equal volumes and concentrations of purified antibodies from SCI or uninjured mice were microinjected into the 
right ventral horn (T12 level) of naive adult WT, $\mathrm{C3}^{-/}$, or $\mathrm{FcRg}^{-/-}$mice. Under anesthesia and using sterile technique, a laminectomy was performed (with partial dural reflection) at the T11-12 vertebral level. To ensure accuracy and to minimize pipette-mediated injury caused by respiratory movement, the spinal column was secured via the spinous processes adjacent to the laminectomy site using Adson forceps fixed in a spinal frame. Sterile glass micropipettes (pulled to an external diameter of 25-30 $\mu \mathrm{m}$ and prefilled with $0.59 \mu \mathrm{g} / 1 \mu \mathrm{l}$ of either SCI or uninjured antibodies dissolved in Pierce antibody purification buffer - a sterile, low-salt buffer solution, $\mathrm{pH}$ 7.2) were positioned approximately $0.3 \mathrm{~mm}$ lateral to the spinal midline using a hydraulic micropositioner (David Kopf Instruments). From the meningeal surface, pipettes were lowered $0.9 \mathrm{~mm}$ into the ventral horn of the underlying gray matter. Using a PicoPump (World Precision Instruments), $1 \mu \mathrm{l}$ of purified $\mathrm{Ab}$ was injected over 15 minutes. Pipettes remained in place for 2 additional minutes to allow the injectate to dissipate into the parenchyma. To facilitate localization of the injection sites, the adjacent dura was marked with sterile charcoal. Postsurgical care, including closing of the surgery site, was performed as described for SCI (see above).

Behavioral evaluation of antibody microinjected animals. Beginning 1 day after microinjection, mice were placed individually in an open field and then subjected to BMS testing (see above). However, because the injections were made into the right ventral horn and therefore affected the right hind limb only, the maximum score allowed for each animal was 5 (frequent or consistent plantar stepping, without forelimb-hind limb coordination). After testing, 15- to 45-second video-clips were taken to further document behavioral differences.

\section{Tissue processing and anatomical analyses}

At 42 or 63 days after SCI or at 7 days after intraspinal microinjection, mice were anesthetized and then transcardially perfused with $25 \mathrm{ml} 0.1 \mathrm{M}$ PBS, followed by $100 \mathrm{ml} 4 \%$ paraformaldehyde in PBS. Brains and spinal cords were removed and post-fixed for 2 hours and then stored in $0.2 \mathrm{M}$ phosphate buffer for 18 hours at $4^{\circ} \mathrm{C}$. The next day, tissues were placed in $30 \%$ sucrose in $0.2 \mathrm{M}$ phosphate buffer and stored for $48-72$ hours. Sucroseinfiltrated tissues were rapidly frozen on powdered dry ice and then stored at $-80^{\circ} \mathrm{C}$. Spinal cord segments spanning the lesion $(1 \mathrm{~cm}$, centered on the impact or injection site) were blocked and embedded in Tissue Tek OCT compound (Sakura Finetechnical Co.) and then molds were rapidly frozen with powdered dry ice. From these blocks, $10 \mu \mathrm{m}$ coronal sections were cut on a cryostat and collected onto SuperfrostPlus slides (Fisher Scientific). SCI and microinjection lesion blocks were collected as 20 sets of serial sections ( $200 \mu \mathrm{m}$ between adjacent sections on each slide) and stored at $-20^{\circ} \mathrm{C}$.

Histology and immunohistochemistry. Adjacent sets of sections encompassing the lesions were stained with eriochrome cyanine (EC) plus cresyl violet (CV) or EC plus anti-mouse 200-kDa neurofilament (chicken anti-neurofilament heavy chain [anti-NFH], see below), as described previously (61). When primary antibodies were derived from mice, nonspecific staining was blocked using a cocktail of bovine serum albumin and complete horse and mouse serum for 1 hour at room temperature. After blocking solution was removed, sections were overlaid with preconjugated primary and secondary antibody cocktail (e.g., containing mouse anti-neurofilament plus biotinlyated horse anti-mouse IgG diluted in blocking solution). This antibody cocktail was incubated for 18 hours at room temperature or $4^{\circ} \mathrm{C}$. In some cases, cell nuclei were revealed using DAPI or Draq5 (Biostatus Limited).
A list of primary antibodies and their final concentrations follows: rat antimouse C1q $(0.133 \mu \mathrm{g} / \mathrm{ml}$, clone 7H8; Abcam), rat anti-mouse CD45R/B220 $(0.83 \mu \mathrm{g} / \mathrm{ml}$, clone RA3-6B2; Serotec), mouse anti-mouse CD68 $(1 \mu \mathrm{g} / \mathrm{ml}$, clone FA-11; Serotec), chicken anti-mouse NFH ( $1 \mu \mathrm{g} / \mathrm{ml}$; Aves Labs), mouse anti-MBP (1:40,000, clone SMI 94, mouse ascites; Covance), goat anti-mouse $\operatorname{IgG}(1 \mu \mathrm{g} / \mathrm{ml}, \gamma$-chain specific; Southern Biotech $), \mathrm{F}\left(\mathrm{ab}^{\prime}\right)_{2}$ fragments of goat anti-mouse IgG heavy and light chains $(1 \mu \mathrm{g} / \mathrm{ml}$; Jackson ImmunoResearch Laboratories Inc.), and goat anti-mouse IgG F( $\left(\mathrm{ab}^{\prime}\right)_{2}$ fragments $(1 \mu \mathrm{g} / \mathrm{ml}$, $\gamma$-chain specific; Jackson ImmunoResearch Laboratories Inc.).

Quantitative spared white matter and lesion analyses. Images of EC plus CVand EC plus NFH-stained sections were digitized using a Zeiss Axioplan II Imaging microscope and an MCID 6.0 Elite system (InterFocus Imaging). High-power digitized sections were printed, and areas of spared white matter, spared gray matter, and lesion were manually circumscribed. Spared white matter was defined as regions containing normal to near-normal densities of both EC and transversely oriented neurofilament staining. Spared grey matter was defined as tissue containing normal gray matter cytoarchitecture with visibly healthy neuron profiles. Lesion was defined as regions lacking either spared white or gray matter. Uniform point grids were placed randomly onto the print outs, and points falling within each area of interest were tallied and recorded. Reference areas for each section (e.g., tissue area) and total tissue volume were estimated in the same manner. Point tallies were converted into volume estimates using the formula, in which volume $=T \times a / p \times{ }^{11} \sum_{i-1} P_{i}$, where $T$ equals the slice spacing, $a / p$ equals the calculated area per point, and ${ }^{11} \sum_{i-1} P_{i}$ equals the sum of the points sampled. Areas per section for each region were calculated using the same formula with omission of the $T$ multiplier.

\section{Statistics}

All data were collected and analyzed without knowledge of group identities. All statistical tests were performed using GraphPad Prism version 5.00 (GraphPad Software). Group means for most analyses were compared using 2-tailed Student's $t$ test or ANOVA with Tukey's post-hoc test. Twoway ANOVA with or without repeated measures and with Bonferroni's post-tests were used to compare data sets containing 2 factors (e.g., behavior and time or area and distance). $P$ values of less than 0.05 were considered statistically significant.

\section{Acknowledgments}

The authors thank Krissy Kigerl, Ming Wang, Violeta McGaughy, and Pat Walters for their intellectual and technical contributions to this work. This work was funded by the following grants from the NIH: NIH R01 NS047175 (to P.G. Popovich), NIH R03 NS055871 (to D.P. Ankeny), and P30-NS045758.

Received for publication May 6, 2009, and accepted in revised form July 8, 2009.

Address correspondence to: Phillip G. Popovich, Center for Brain and Spinal Cord Repair, The Ohio State University College of Medicine, 786 Biomedical Research Tower, 460 West 12th Avenue, Columbus, Ohio 43210-1239, USA. Phone: (614) 688-8576; Fax: (614) 688-5463; E-mail: phillip.popovich@osumc.edu.

\footnotetext{
1. Kigerl, K.A., McGaughy, V.M., and Popovich, P.G. 2006. Comparative analysis of lesion development and intraspinal inflammation in four strains of mice following spinal contusion injury. J. Comp. Neurol. 494:578-594.

2. Popovich, P.G., et al. 1999. Depletion of hematogenous macrophages promotes partial hindlimb recovery and neuroanatomical repair after experimental
}

spinal cord injury. Exp. Neurol. 158:351-365

3. Sroga, J.M., Jones, T.B., Kigerl, K.A., McGaughy, V.M., and Popovich, P.G. 2003. Rats and mice exhibit distinct inflammatory reactions after spinal cord injury. J. Comp. Neurol. 462:223-240.

4. Blight, A.R. 1985. Delayed demyelination and macrophage invasion: a candidate for secondary cell damage in spinal cord injury. Cent. Nerv. Syst. Trauma.
2:299-315.

5. Blight, A.R. 1994. Effects of silica on the outcome from experimental spinal cord injury: implication of macrophages in secondary tissue damage. Neuroscience. 60:263-273.

6. Carlson, S.L., Parrish, M.E., Springer, J.E., Doty, K., and Dossett, L. 1998. Acute inflammatory response in spinal cord following impact injury. Exp. Neurol. 
151:77-88.

7. Dusart, I., and Schwab, M.E. 1994. Secondary cell death and the inflammatory reaction after dorsal hemisection of the rat spinal cord. Eur. J. Neurosci. 6:712-724.

8. Fleming, J.C., et al. 2006. The cellular inflammatory response in human spinal cords after injury. Brain. 129:3249-3269.

9. Zhang, Z., Krebs, C.J., and Guth, L. 1997. Experimental analysis of progressive necrosis after spinal cord trauma in the rat: etiological role of the inflammatory response. Exp. Neurol. 143:141-152.

10. Hayes, K.C., et al. 2002. Elevated serum titers of proinflammatory cytokines and CNS autoantibodies in patients with chronic spinal cord injury. J. Neurotrauma. 19:753-761.

11. Mizrachi, Y., et al. 1983. Systemic humoral factors participating in the course of spinal cord injury. Paraplegia. 21:287-293.

12. Kil, K., et al. 1999. T-cell responses to myelin basic protein in patients with spinal cord injury and multiple sclerosis. J. Neuroimmunol. 98:201-207.

13. Ankeny, D.P., Lucin, K.M., Sanders, V.M. McGaughy, V.M., and Popovich, P.G. 2006. Spinal cord injury triggers systemic autoimmunity: evidence for chronic B lymphocyte activation and lupus-like autoantibody synthesis. J. Neurochem. 99:1073-1087.

14. Ankeny, D.P., and Popovich, P.G. 2009. Mechanisms and implications of adaptive immune responses after traumatic spinal cord injury. Neuroscience. 158:1112-1121.

15. Schlachetzki, F., Zhu, C., and Pardridge, W.M. 2002. Expression of the neonatal $\mathrm{Fc}$ receptor $(\mathrm{FcRn})$ at the blood-brain barrier. J. Neurochem. 81:203-206.

16. Popovich, P.G., Horner, P.J., Mullin, B.B., and Stokes, B.T. 1996. A quantitative spatial analysis of the blood-spinal cord barrier I. Permeability changes after experimental spinal contusion injury. Exp. Neurol. 142:258-275.

17. Noble, L.J., and Wrathall, J.R. 1989. Distribution and time course of protein extravasation in the rat spinal cord after contusive injury. Brain Res. 482:57-66.

18. Gupta, R.K., and Siber, G.R. 1995. Method for quantitation of $\operatorname{IgG}$ subclass antibodies in mouse serum by enzyme-linked immunosorbent assay. J. Immunol. Methods. 181:75-81.

19. Upender, M.B., and Naegele, J.R. 1999. Activation of microglia during developmentally regulated cell death in the cerebral cortex. Dev. Neurosci. 21:491-505

20. Abdul-Majid, K.B., et al. 2002. Fc receptors are critical for autoimmune inflammatory damage to the central nervous system in experimental autoimmune encephalomyelitis. Scand. J. Immunol. 55:70-81.

21. Griot-Wenk, M., Griot, C., Pfister, H., and Vandevelde, M. 1991. Antibody-dependent cellular cytotoxicity in antimyelin antibody-induced oligodendrocyte damage in vitro. J. Neuroimmunol. 33:145-155.

22. Linington, C., et al. 1989. The role of complement in the pathogenesis of experimental allergic encephalomyelitis. Brain. 112:895-911.

23. Linington, C., Lassmann, H., Morgan, B.P., and Compston, D.A. 1989. Immunohistochemical localisation of terminal complement component C9 in experimental allergic encephalomyelitis. Acta Neuropathol. (Berl.). 79:78-85.

24. Mosley, K., and Cuzner, M.L. 1996. Receptor-mediated phagocytosis of myelin by macrophages and microglia: effect of opsonization and receptor blocking agents. Neurochem. Res. 21:481-487.

25. Beuche, W., and Friede, R.L. 1986. Myelin phagocytosis in Wallerian degeneration of peripheral nerves depends on silica-sensitive, $\mathrm{bg} / \mathrm{bg}$-negative and FC- positive monocytes. Brain Res. 378:97-106.

26. Chouchakova, N., et al. 2001. Fc gamma RIII-mediated production of TNF-alpha induces immune complex alveolitis independently of CXC chemokine generation. J. Immunol. 166:5193-5200.

27. Jones, T.B., et al. 2002. Pathological CNS autoimmune disease triggered by traumatic spinal cord injury: implications for autoimmune vaccine therapy. J. Neurosci. 22:2690-2700.

28. Popovich, P.G., and Longbrake, E.E. 2008. Can the immune system be harnessed to repair the CNS? Nat. Rev. Neurosci. 9:481-493.

29. Hirschberg, D.L., Yoles, E., Belkin, M., and Schwartz, M. 1994. Inflammation after axonal injury has conflicting consequences for recovery of function: rescue of spared axons is impaired but regeneration is supported. J. Neuroimmunol. 50:9-16.

30. Zhang, M., Alicot, E.M., and Carroll, M.C. 2008. Human natural IgM can induce ischemia/reperfusion injury in a murine intestinal model. $\mathrm{Mol}$. Immunol. 45:4036-4039.

31. Zhang, M., et al. 2006. Activation of the lectin pathway by natural IgM in a model of ischemia/reperfusion injury. J. Immunol. 177:4727-4734.

32. Austen, W.G., Jr., et al. 2004. Murine hindlimb reperfusion injury can be initiated by a self-reactive monoclonal IgM. Surgery. 136:401-406.

33. Lucin, K.M., et al. 2007. Impaired antibody synthesis after spinal cord injury is level dependent and is due to sympathetic nervous system dysregulation. Exp. Neurol. 207:75-84.

34. Leinhase, I., et al. 2006. Reduced neuronal cell death after experimental brain injury in mice lacking a functional alternative pathway of complement activation. BMC Neurosci. 7:55.

35. Galvan, M.D., et al. 2008. Deficiency in complement $\mathrm{C} 1 \mathrm{q}$ improves histological and functional locomotor outcome after spinal cord injury. J. New rosci. 28:13876-13888.

36. Nguyen, H.X., Galvan, M.D., and Anderson, A.J 2008. Characterization of early and terminal complement proteins associated with polymorphonuclear leukocytes in vitro and in vivo after spinal cord injury. J. Neuroinflammation. 5:26.

37. Qiao, F., et al. 2006. Complement plays an important role in spinal cord injury and represents a therapeutic target for improving recovery following trauma. Am. J. Pathol. 169:1039-1047.

38. Simpson, J., et al. 1986. Autoantibodies to Alzheimer and normal brain structures from virus-transformed lymphocytes. J. Neuroimmunol. 13:1-8.

39. Bruck, W., and Friede, R.L. 1991. The role of complement in myelin phagocytosis during PNS wallerian degeneration. J. Neurol. Sci. 103:182-187.

40. Servettaz, A., et al. 2008. Natural anti-endothelial cell antibodies. Autoimmun. Rev. 7:426-430.

41. Anderson, A.J., Robert, S., Huang, W., Young, W., and Cotman, C.W. 2004. Activation of complement pathways after contusion-induced spinal cord injury. J. Neurotrauma. 21:1831-1846.

42. Vincent, A., Lang, B., and Kleopa, K.A. 2006. Autoimmune channelopathies and related neurological disorders. Neuron. 52:123-138.

43. Davies, A.L., Hayes, K.C., and Dekaban, G.A. 2007. Clinical correlates of elevated serum concentrations of cytokines and autoantibodies in patients with spinal cord injury. Arch. Phys. Med. Rehabil. 88:1384-1393.

44. Genain, C.P., Cannella, B., Hauser, S.L., and Raine, C.S. 1999. Identification of autoantibodies associated with myelin damage in multiple sclerosis. Nat. Med. 5:170-175.

45. Raine, C.S., Cannella, B., Hauser, S.L., and Genain, C.P. 1999. Demyelination in primate autoimmune encephalomyelitis and acute multiple sclerosis lesions: a case for antigen-specific antibody mediation. Ann. Neurol. 46:144-160.

46. Mehta, P.D., Mehta, S.P., and Patrick, B.A. 1982. Identification of light chain type bands in CSF and serum oligoclonal IgG from patients with multiple sclerosis. J. Neuroimmunol. 2:119-129.

47. Elkon, K., and Casali, P. 2008. Nature and functions of autoantibodies. Nat. Clin. Pract. Rheumatol. 4:491-498.

48. Lacroix-Desmazes, S., et al. 1998. Self-reactive antibodies (natural autoantibodies) in healthy individuals. J. Immunol. Methods. 216:117-137.

49. Carroll, M.C., and Holers, V.M. 2005. Innate autoimmunity. Adv. Immunol. 86:137-157.

50. Hulse, R.E., Swenson, W.G., Kunkler, P.E., White, D.M., and Kraig, R.P. 2008. Monomeric IgG is neuroprotective via enhancing microglial recycling endocytosis and TNF-alpha. J. Neurosci. 28:12199-12211.

51. Samuelsson, A., Towers, T.L., and Ravetch, J.V. 2001. Anti-inflammatory activity of IVIG mediated through the inhibitory Fc receptor. Science. 291:484-486.

52. Asakura, K., Miller, D.J., Pease, L.R., and Rodriguez, M. 1998. Targeting of IgMkappa antibodies to oligodendrocytes promotes CNS remyelination. J. Neurosci. 18:7700-7708.

53. Miller, D.J., Sanborn, K.S., Katzmann, J.A., and Rodriguez, M. 1994. Monoclonal autoantibodies promote central nervous system repair in an animal model of multiple sclerosis. J. Neurosci. 14:6230-6238.

54. Rodriguez, M., and Lennon, V.A. 1990. Immunoglobulins promote remyelination in the central nervous system. Ann. Neurol. 27:12-17.

55. Vani, J., et al. 2008. Role of natural antibodies in immune homeostasis: IVIg perspective. Autoimmun. Rev. 7:440-444.

56. Kazatchkine, M.D., and Kaveri, S.V. 2001. Immunomodulation of autoimmune and inflammatory diseases with intravenous immune globulin. N. Engl. J. Med. 345:747-755.

57. Lee, C.H., Suh, C.H., Lee, J., Kim, Y.T., and Lee, S.K. 2003. The effects of anti-idiotypic antibody on antibody production and apoptosis of anti-dsDNA antibody producing cells. Clin. Exp. Rheumatol. 21:291-300.

58. Kitamura, D., Roes, J., Kuhn, R., and Rajewsky, K. 1991. A B cell-deficient mouse by targeted disruption of the membrane exon of the immunoglobulin mu chain gene. Nature. 350:423-426.

59. Jakeman, L.B., et al. 2000. Traumatic spinal cord injury produced by controlled contusion in mouse. J. Neurotrauma. 17:299-319.

60. Basso, D.M., et al. 2006. Basso mouse scale for locomotion detects differences in recovery after spinal cord injury in five common mouse strains. J. Neurotrauma. 23:635-659.

61. Kigerl, K.A., et al. 2007. Toll-like receptor (TLR)-2 and TLR-4 regulate inflammation, gliosis, and myelin sparing after spinal cord injury. J. Neurochem. 102:37-50. 